\title{
Incremental Role of Pancreatic Magnetic Resonance Imaging after Staging Computed Tomography to Evaluate Patients with Pancreatic Ductal Adenocarcinoma
}

\author{
Hye Jin Kim, MD ${ }^{1, a}$ \\ Mi-Suk Park, MD \\ Jin Yong Lee, MD \\ Kyunghwa Han, $\mathrm{PhD}^{2}$ \\ Yong Eun Chung, MD'1 \\ Jin-Young Choi, MD ${ }^{1}$ \\ Myeong-Jin Kim, MD \\ Chang Moo Kang, MD
}

\begin{abstract}
${ }^{1}$ Department of Radiology and Research Institute of Radiological Science, Severance Hospital, Yonsei University College of Medicine, Seoul, ${ }^{2}$ Department of Radiology, Yonsei Biomedical Research Institute, Research Institute of Radiological Science, Severance Hospital, Yonsei University College of Medicine, Seoul, ${ }^{3}$ Department of Surgery, Severance Hospital, Yonsei University College of Medicine, Seoul, Korea
\end{abstract}

\section{Correspondence: Mi-Suk Park, MD}

Department of Radiology and Research Institute of Radiological Science, Severance Hospital,

Yonsei University College of Medicine,

50-1 Yonsei-ro, Seodaemun-gu, Seoul 03722, Korea

Tel: 82-2-2008-7400

Fax: 82-2-393-3035

E-mail: RADPMS@yuhs.ac

Received August 26, 2017

Accepted February 4, 2018

Published Online February 5, 2018

aPresent address: Department of Radiology, Ajou University School of Medicine, Suwon, Korea

\begin{abstract}
Purpose
The purpose of this study was to investigate the impact of contrast enhanced pancreatic magnetic resonance imaging (MRI) in resectability and prognosis evaluation after staging computed tomography (CT) in patients with pancreatic ductal adenocarcinoma (PDA).
\end{abstract}

\section{Materials and Methods}

From January 2005 to December 2012, 298 patients were diagnosed to have potentially resectable stage PDA on CT. Patients were divided into CT+MR (patients underwent both $\mathrm{CT}$ and MRl; $n=216$ ) and $C T$ only groups ( $n=82$ ). Changes in resectability staging in the $C T+M R$ group were evaluated. The overall survival was compared between the two groups. The recurrence-free survival and median time to liver metastasis after curative surgery were compared between the two groups.

\section{Results}

Staging was changed from resectable on CT to unresectable state on MRI in 14.4\% of (31 of 216 patients) patients of the CT+MR group. The overall survival and recurrence-free survival rates were not significantly different between the two groups $(p=0.162$ and $p=0.721$, respectively). The median time to liver metastases after curative surgery in the CT+MR group (9.9 months) was significantly longer than that in the CT group ( 4.2 months) $(p=0.011)$.

\section{Conclusion}

Additional MRI resulted in changes of resectability and treatment modifications in a significant proportion of patients who have potentially resectable state at CT and in prolonged time to liver metastases in patients after curative surgery. Additional MRI to standard staging CT can be recommended for surgical candidates of PDA.

Key words

Pancreas ductal adenocarcinoma, Computed tomography, Magnetic resonance imaging, Resectability

\section{Introduction}

Pancreatic ductal adenocarcinoma (PDA) is one of the greatest challenging tumors among malignant neoplasms because of its aggressive course with persistent high cancer related mortality rate over the last few decades [1]. The 5 -year survival rate in patients with pancreatic cancer is less than 5 to $6 \%$ worldwide [2]. Surgical resection is the only potential for curative treatment and can prolong survival, but only $15 \%$ to $25 \%$ of patients constitute the surgical candidates at the time of diagnosis $[1,3,4]$. Even after curative surgery, the actuarial 5-year overall survival has been reported to be only $18 \%$ to $25 \%$ in the most experienced of hands and in-hospital mortality was reported from $4.6 \%$ to $7.8 \%[1,5]$. These facts emphasize the need to avoid futile sur- 
gery to minimize perioperative mortality and to improve patients' quality of life and long-term survival.

To date, computed tomography (CT) has become a widely accepted imaging modality as the standard for patients with clinical suspicion of pancreatic cancer [3,6-8]. However, several studies have shown that $\mathrm{CT}$ as a sole imaging modality is insufficient in the determination of surgical unresectability of PDA [9-11]. A previous study with meta-analysis reported that the mean probability of unresectable disease after CT scan across studies was approximately $61 \%$ in patients with potentially resectable pancreatic and periampullary cancer [9]. With recent advances in magnetic resonance imaging (MRI), MRI has been widely used to evaluate hepatopancreaticobiliary disease. However, it is not clear as to which pancreatic cancer patients should undergo MRI in addition to the standard pancreatic CT. National Comprehensive Cancer Network (NCCN) clinical practice guidelines ver. 2. 2015 proposed that contrast enhanced MRI with magnetic resonane (MR) cholangiopancreatography (MRCP) can be considered to further evaluate patients in whom no mass was detected on pancreatic protocol CT or those with CT-indeterminate liver lesions [12]. This recommendation might have been based on the fact that MRI is more sensitive in detecting small masses or minimal ductal changes in the pancreas and small lesions in the liver owing to its high tissue contrast [7]. However, there is no existing clear guideline whether the MRI in the surgical candidates with visible pancreatic mass on CT is additionally needed or not. Considering the high perioperative mortality and low survival after curative surgery in patients with PDA, the role of imaging in PDA should be further emphasized with regard to accurate staging for resectability to minimize the morbidity from unnecessary laparotomies or major surgeries, in addition to maximize the survival benefit in patients with curative treatment. To the best of our knowledge, there has been no previous study demonstrating the incremental value of the additional MRI performed after CT, especially in predicting the resectability and the clinical outcome.

In this study, we aimed to investigate the clinical impact of MRI on resectability evaluation and the effect on the overall and recurrence-free survival in patients with standard CT initially interpreted to have potentially resectable PDA.

\section{Materials and Methods}

\section{Patients}

We searched the electrical medical records at out institution to identify the study patients. The eligibility criteria for the main investigation included the following: (1) patients who were histopathologically diagnosed with PDA between January 2005 and December 2012; (2) patients identified to have potentially resectable PDA on CT. One thousand eight hundred fifty-two consecutive patients were newly diagnosed with PDA during this period. All of them underwent CT imaging for work-up of PDA. Our standardized clinical reports on staging CT for PDA, which were made by experienced board-certified abdominal radiologists, were used to identify relevant patients. Two radiologists in consensus reviewed the imaging findings of the baseline CT with reports, and subsequently classified PDA patients into four categories (resectable, borderline resectable, unresectable, and indeterminate state) based on the 2015 NCCN practice guidelines, in order to determine the surgical resectability [12]. The potentially resectable PDA in our study included resectable, borderline resectable, and indeterminate state. Patients were excluded if they had the following: (1) incomplete clinical or radiological data $(n=566),(2)$ unresectable state on CT imaging review $(n=946),(3)$ non-contrast CT or $\operatorname{MRI}(n=22)$, and (4) age more than 80 years $(n=20)$. Hence, a total of 298 patients (165 men, 133 women; mean age, 62 years; range, 27 to 80 years) were included in the main study population with potentially resectable PDA on CT. Of these, 82 patients only underwent CT (CT group) and 216 patients underwent MRI within 4 weeks after CT acquisition (CT+MR group). The recommendation regarding the subsequent MRI was made in consensus at multidisciplinary pancreatobiliary meeting, but the final decision was left to the attending physician's judgment. Clinical information including patient demographics, laboratory data, treatment methods, and histopathology reports were reviewed using electronic medical records. Of the 298 patients, 161 (54\%) who received curative surgical resection were included for subgroup analysis to evaluate the recurrence-free survival and time to liver metastasis after surgery. The flowchart of the study population is presented in Fig. 1.

\section{CT imaging}

All patients underwent CT examinations with either a 16-channel or 64-channel scanner (Sensation 16 or Sensation 64, Siemens, Erlangen, Germany; Brilliance 64, Philips Healthcare, Cleveland, $\mathrm{OH}$ ). After obtaining non-contrast CT images, contrast-enhanced pancreatic CT were performed with intravenous administration of nonionic contrast medium (Ultravist 300, Schering, Berlin, Germany). Pancreatic and portal venous phase imaging was obtained by adding 18 seconds to the time of peak abdominal aortic enhancement calculated at the hepatic hilum, and 18 seconds to the end of the pancreatic phase, respectively. In 38 patients, only the portal venous phase $\mathrm{CT}$ was obtained. The scanning parameters 


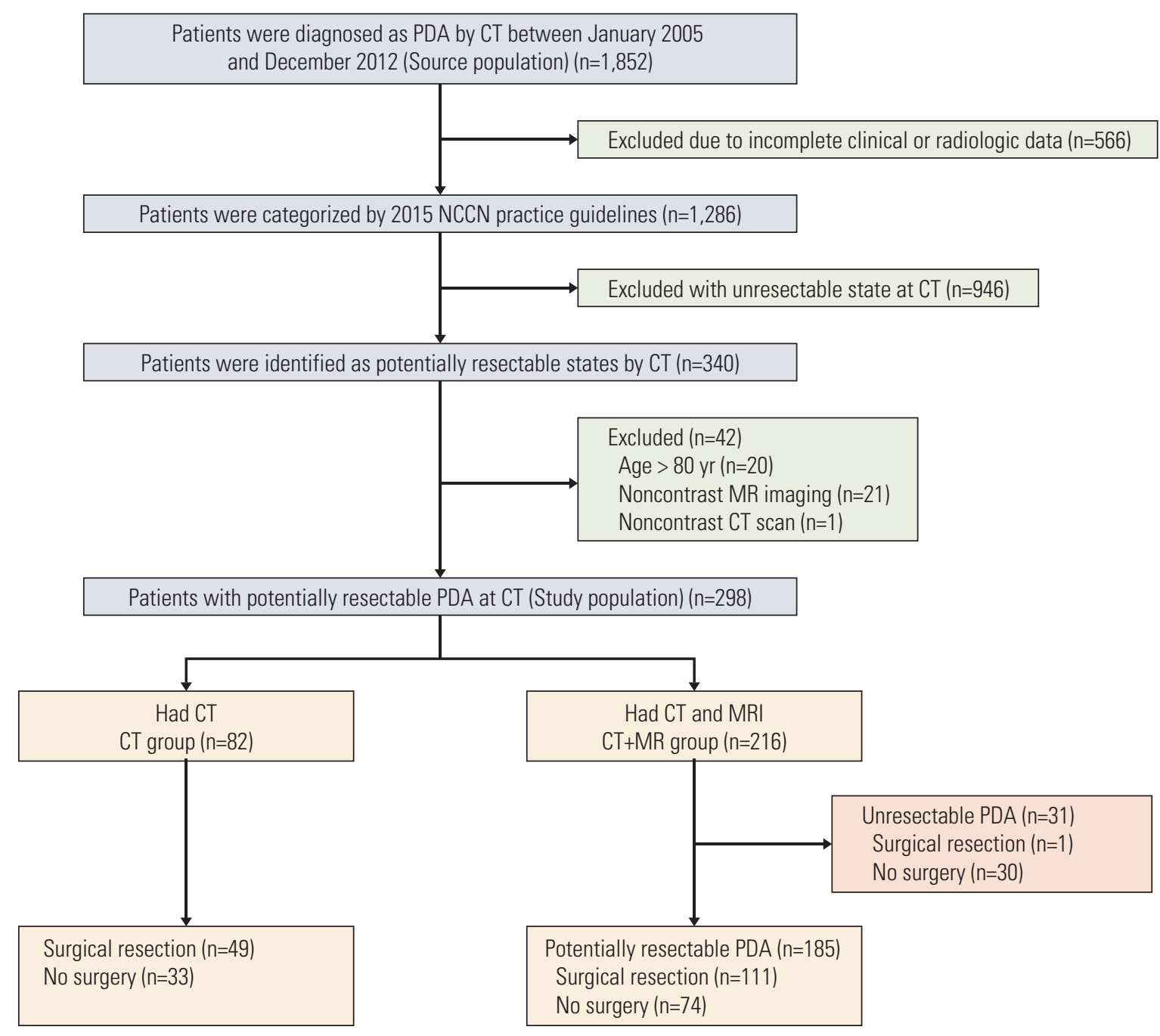

Fig. 1. Flow diagram of study population. PDA, pancreas ductal adenocarcinoma; $\mathrm{CT}$, computed tomography; NCCN, National Comprehensive Cancer Network; MRI, magnetic resonance imaging.

were as follows: beam collimation, 0.75 and $0.625 \mathrm{~mm}$; slice thickness, 5 and $3 \mathrm{~mm}$; reconstruction interval, 5 and $3 \mathrm{~mm}$; rotation time, 0.5 seconds; effective tube current-time charge, 150-250 mAs; $120 \mathrm{kVp}$ for the 16- and 64-channel scanner, respectively.

\section{Magnetic resonance imaging}

MRI was performed by using a 1.5-T (Intera Achieva, Philips Medical Systems, Best, Netherlands) or 3.0-T scanner (Magnetom Trio Tim, Siemens Medical Solutions, Erlangen, Germany) using 4- or 16-channel torso-array coil. All patients underwent baseline non-contrast and post-contrast dynamic MRI. Both single-section and navigator-triggered MRCP imaging were performed. Dynamic T1-weighted imaging was obtained after administering one of the two contrast materials (Primovist, Bayer Schering Pharma, Berlin, Germany; Dotarem, Guerbet, France): a bolus injection of 0.1 $\mathrm{mL} / \mathrm{kg}$ gadoxetic acid $(\mathrm{n}=186)$ at a rate of $1.0 \mathrm{~mL} / \mathrm{sec}$ or 0.2 $\mathrm{mL} / \mathrm{kg}$ gadoterate meglumine $(\mathrm{n}=30)$ at a rate of $2.0 \mathrm{~mL} / \mathrm{sec}$, followed by a $20 \mathrm{~mL}$ saline flush using a power injector. The peak time of the time-density curve to determine image acquisition timing was achieved using a test-bolus technique in which $1 \mathrm{~mL}$ of gadoxetic acid or of gadoterate meglumine was injected with a saline flush. The arterial phase began 2 or 3 seconds after the peak aortic enhancement was determined. Subsequent portal (50 seconds) and transitional ( 3 minutes) phase images were obtained. Additional hepatobiliary phase (20 minutes) images were obtained when gadoxetic acid (hepatocyte-specific agent) were used. Diffu- 
sion-weighted imaging was obtained at $b$ values of $0,50,400$, and $800 \mathrm{sec} / \mathrm{mm}^{2}$. In our institution, both hepatocyte-specific contrast agent (gadoxetic acid) and diffusion weighted imaging (DWI) were introduced on February 2008. Thereafter, they became the routine protocol. Detailed MR parameters are provided in S1 Table.

\section{Assessment of resectability}

Two, board-certified abdominal radiologists (with 6 and 11 years of experience in abdominal imaging, respectively) in consensus retrospectively reviewed the all baseline CT images ( $n=298$ patients) with reference to prospectively interpreted reports at Picture Archiving Communication System. They classified the patients into three categories (resectable, borderline resectable, and unresectable states) based on the 2015 NCCN, as defined in S2 Table. Patients who did not belong to above the three NCCN categories (i.e., patients with hepatic lesions considered too small to characterize or lesions which are difficult to categorize between benign or malignant lesions in any organ) were classified as an indeterminate state. After four weeks, the same radiologists reviewed and classified the MRI ( $\mathrm{n}=216$ patients) in the same manner as CT. While reviewing the MRI, they simultaneously referred to the CT imaging findings as in routine clinical practice. The radiologists were informed that all patients had PDA, but they were blinded to all other things, including clinical and laboratory findings and the histo-pathological stage.

\section{Outcomes and follow-up evaluation}

The primary outcome of this study was change in surgical resectability after additional MRI in patients with potentially resectable PDA on CT. Secondary outcomes included overall survival rates for all patients, and recurrence-free survival rates and time to liver metastases for patients receiving curative surgical treatment, between the CT only and CT+MR groups. The index date was defined as the date on which the patient underwent baseline CT for the diagnosis of PDA. Patients were followed up from the index date till death or the last follow-up date. Each patient's overall and recurrencefree survival was calculated from the index date till death, date of last follow-up evaluation, or confirmation of recurrence. To substantiate the completeness of the follow-up data, information about vital status was obtained from the National Population Registry of the Korea National Statistical Office using unique personal identification numbers. Curative-intent treatments were defined as curative surgical resection regardless of neoadjuvant concurrent chemoradiotherapy. The routine follow-up protocol included serum carbohydrate antigen (CA) 19-9 and contrast-enhanced CT.
Recurrence of PDA was defined to have occurred when local recurrence or distant metastatic lesions were first evident in the medical record, based on a combination of follow-up CT imaging, CA 19-9 level, histological confirmation, or physical examination. Time to liver metastases was calculated from the date of curative surgery to the initial presentation date of liver metastases on follow-up CT among patients receiving curative surgical treatment. Subgroup analysis was performed for patients in the CT+MR group. They were divided into persistently potentially resectable and unresectable states based on the CT+MR findings, and the overall and recurrent-free survival was compared between the two groups.

\section{Statistical analysis}

Clinical characteristics of all patients were compared between the CT only and CT+MR groups using the Student $\mathrm{t}$ test for continuous variables and the chi-square test for categorical variables. Kaplan-Meier survival analysis and Cox proportional hazards regression were used to assess the impact of additional MRI on overall survival and recurrencefree survival. Time to liver metastases for the CT and CT+MR groups were compared by using Mann-Whitney U test.

Statistical analyses were performed by our statistician using the R software ver. 3.3.1. (R Foundation for Statistical Computing, Vienna, Austria). All reported p-values were two sided with a significant level of less than 0.05 .

\section{Ethical statement}

This historical cohort study was approved by the institutional review board of Severance Hospital (IRB No. 20161611) that is a tertiary referral center with high volume of pancreatic cancer, and the requirement for informed consent from patients was waived.

\section{Results}

\section{Patient characteristics}

Clinical characteristics of the patients are summarized in Table 1. The proportion of patients receiving neoadjuvant concurrent chemoradiotherapy before surgical resection was significantly higher in the CT group (53.1\%) than in the $\mathrm{CT}+\mathrm{MR}$ group $(30.4 \%)(\mathrm{p}=0.006)$. The other baseline characteristics of patients in the CT and CT+MR groups were not significantly different. The proportion of patients undergoing additional MRI was 72.4\% (142 of 196 patients) for the 
Table 1. Characteristics of the full cohort

\begin{tabular}{|c|c|c|c|c|}
\hline Characteristic & Total $(n=298)$ & $\mathrm{CT}(\mathrm{n}=82)$ & $\mathrm{CT}+\mathrm{MR}(\mathrm{n}=216)$ & p-value \\
\hline Age (yr) & $62.19 \pm 10.02$ & $61.94 \pm 10.64$ & $62.28 \pm 9.79$ & 0.795 \\
\hline Male sex & $164(55)$ & $48(58.5)$ & $116(53.7)$ & 0.454 \\
\hline \multicolumn{5}{|l|}{ Associated factor } \\
\hline Smoking & $79(26.5)$ & $20(24.4)$ & $59(27.3)$ & 0.610 \\
\hline Alcohol & $80(26.8)$ & $21(25.6)$ & $59(27.3)$ & 0.767 \\
\hline Diabetes & $110(36.9)$ & $35(42.7)$ & $75(34.7)$ & 0.204 \\
\hline CA19-9 level (U/mL) & $213(43.1-87.9)$ & $271(63.1-921)$ & $183(38.5-871)$ & 0.275 \\
\hline \multicolumn{5}{|l|}{ CT phases } \\
\hline Single & $38(12.8)$ & $8(9.8)$ & $30(13.9)$ & 0.370 \\
\hline Dual & $260(87.2)$ & $74(90.2)$ & $186(86.1)$ & \\
\hline Curative treatment & $161(54.0)$ & $49(59.8)$ & $112(51.9)$ & 0.221 \\
\hline Surgical resection without neoadjuvant CCRT & $101(62.7)$ & $23(46.9)$ & $78(69.6)$ & 0.006 \\
\hline Surgical resection after neoadjuvant CCRT & $60(37.3)$ & $26(53.1)$ & $34(30.4)$ & \\
\hline \multicolumn{5}{|l|}{ Resected margin } \\
\hline R0 & $144(89.4)$ & $42(85.7)$ & $102(91.1)$ & 0.309 \\
\hline R1 & $17(10.6)$ & $7(14.3)$ & $10(8.9)$ & \\
\hline Follow-up period (yr) & $1.49(0.85-3.20)$ & $1.59(0.91-4.57)$ & $1.43(0.82-2.79)$ & 0.193 \\
\hline
\end{tabular}

Values are presented as mean $\pm \mathrm{SD}$, number $(\%)$, or median $(\mathrm{IQR})$. Values were evaluated by chi-square test for categorical variables and by independent $\mathrm{t}$ test for continuous variables. $\mathrm{CT}$, computed tomography; $\mathrm{CT}+\mathrm{MR}$, patients underwent both CT and magnetic resonance imaging; CA19-9, carbohydrate antigen 19-9; CCRT, concurrent chemoradiotherapy; R0, negative resection margin; R1, positive microscopic resection margin.

Table 2. Changes in the surgical resectability of pancreatic ductal adenocarcinoma by MRI at CT+MR group

\begin{tabular}{lcccccc} 
& & \multicolumn{5}{c}{ MR assessment of surgical resectability } \\
\cline { 3 - 7 } CT assessment & Sub-total & Resectable & $\begin{array}{c}\text { Borderline } \\
\text { resectable }\end{array}$ & Indeterminate & Unresectable & $\begin{array}{c}\text { Changes in } \\
\text { surgical } \\
\text { resectability }\end{array}$ \\
Resectable & $142(65.7)$ & 123 & 8 & 0 & $11(7.7)$ & $11(7.7)$ \\
Borderline resectable & $49(22.7)$ & 4 & 33 & 0 & $12(24.5)$ & $12(24.5)$ \\
Indeterminate & $25(11.6)$ & 8 & 6 & 3 & $8(32.0)$ & $8(32.0)$ \\
Total & $216(100)$ & $135(62.5)$ & $47(21.8)$ & $3(1.4)$ & $31(14.4)$ & $31(14.4)$ \\
\hline
\end{tabular}

Values are presented as number (\%). MRI, magnetic resonance imaging; $\mathrm{CT}+\mathrm{MR}$, patients underwent both computed tomography and magnetic resonance imaging; CT, computed tomography.

resectable state, $66.2 \%$ (49 of 74 patients) for the borderline resectable state, and $89.3 \%$ ( 25 of 28 patients) for the indeterminate state at $\mathrm{CT}$ classification, without statistical difference $(\mathrm{p}=0.067)$.

\section{Changes in surgical resectability and treatment of PDA after additional MR imaging}

Of the 216 patients in the CT+MR group, a total of 31 patients $(14.4 \%)$ who had potentially resectable states on CT were changed to have unresectable state on additional MRI (Table 2). Liver metastases $(n=16)$, major vascular invasion $(n=13)$, enlarged para-aortic lymph node $(n=1)$, and peritoneal seeding $(\mathrm{n}=1)$ were additionally found by MRI. Of the 16 patients with liver metastases diagnosed on MRI, nine patients had negative-liver-on-CT $(4.7 \%, 9$ of 191 resectable or borderline resectable group on CT) (Fig. 2) and seven patients had diminutive indeterminate hepatic lesions on CT $(35 \%, 7$ of 20). Of the 13 patients with unresectable state due to major vascular invasion on MRI, three patients had no 

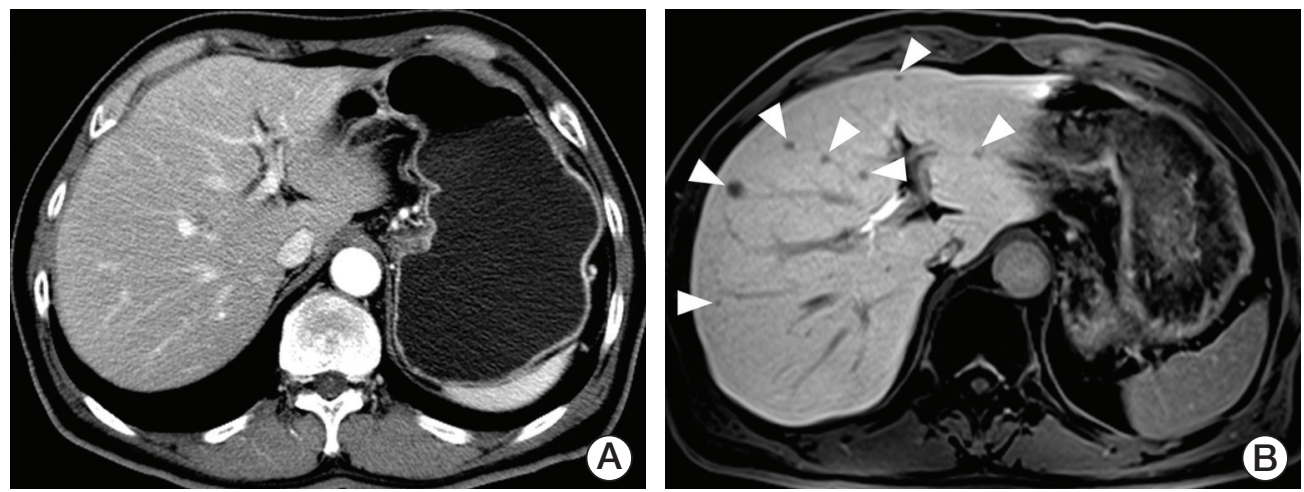

Fig. 2. Computed tomography (CT) and magnetic resonance (MR) images obtained in a 65-year-old man with pancreas ductal adenocarcinoma. (A, B) Portal phase CT image demonstrates no focal liver lesion (A). This patient was classified into surgically resectable state at CT (pancreas cancer not seen at this image). However, additional gadoxetic acid-enhanced MR image shows multiple subcentimeter low signal intensity nodules indicating hepatic metastases at hepatobiliary phase (arrowheads) (B). Finally, this patient was reclassified into unresectable state after MR imaging.

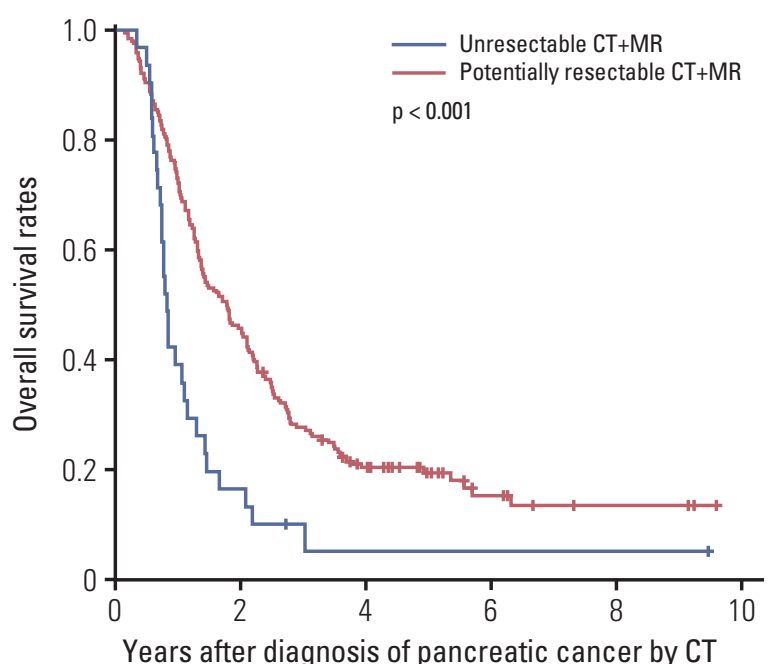

No. at risk

$\begin{array}{rrrrrrr}\text { PR CT+MR } & 185 & 84 & 33 & 10 & 4 & 0 \\ \text { UR CT+MR } & 31 & 5 & 1 & 1 & 1 & 1\end{array}$

Fig. 3. Overall survival of patients belonged to the potentially resectable (PR) CT $+\mathrm{MR}$ group (patients underwent both computed tomography and magnetic resonance imaging) and the unresectable (UR) CT+MR group.

major vessel invasion on CT $(1.4 \%, 2$ of 142 resectable group and $4 \%, 1$ of 25 indeterminate group on CT) and 10 patients had borderline vascular invasion on CT (20.4\%, 10 of 49 borderline group on CT). Two of five patients with indeterminate extrahepatic lesions (omental haziness and preaortic lymph node) other than indeterminate hepatic lesions on CT were changed to surgically resectable state after MRI. After analysis of MRI, 185 patients in CT+MR group were remained as persistently potentially resectable. There was no significant difference in the overall proportion of the patients who received curative surgical treatment between the CT only $(49$ of $82,59.8 \%)$ and the persistently potentially resectable CT+MR groups $(111 / 185,60 \%)(p=0.970)$. Thirty of 31 patients reclassified into the unresectable state after MRI did not receive curative surgical treatment $(96.8 \%)$.

\section{Overall and recurrence-free survival}

The median follow-up period was 1.6 years for the CT group and 1.4 years for the $\mathrm{CT}+\mathrm{MR}$ group without significant difference between the two groups ( $\mathrm{p}=0.193)$. The 5-year overall survival rates of the CT only and CT+MR groups were $24.1 \%$ and $17.2 \%$, respectively, without statistical difference ( $p=0.162)$ (S3A Fig.).

Within the CT+MR group, the 5-year overall survival rates between the potentially resectable $(19.2 \%)$ and unresectable $(4.8 \%)$ subgroups were significantly different $(p<0.001)$ (hazard ratio [HR], 2.27; 95\% confidence interval [CI], 1.53 to 3.37; $\mathrm{p}<0.001$ ) (Fig. 3). The curative surgical treatment performed in PDA patients was significantly associated with a lower risk of overall patient mortality (HR, 0.39; 95\% CI, 0.29 to $0.52 ; \mathrm{p}<0.001$ ) (Table 3).

A total of 161 patients (54\%) received curative surgical resection with 112 patients in the $\mathrm{CT}+\mathrm{MR}$ group and 49 patients in the CT group. Five-year recurrence-free survival rates were $29.8 \%$ and $29.7 \%$, respectively, without statistical difference $(p=0.721)$ (S3B Fig.). However, the median time to 
Table 3. Predictive factors of overall patient mortality and PDA recurrence in the full cohort

\begin{tabular}{|c|c|c|c|c|c|c|}
\hline \multirow{2}{*}{ Variable } & \multicolumn{3}{|c|}{ Univariate analysis } & \multicolumn{3}{|c|}{ Multivariate analysis } \\
\hline & HR & $95 \% \mathrm{CI}$ & p-value & $\overline{\text { HR }}$ & $95 \% \mathrm{CI}$ & p-value \\
\hline \multicolumn{7}{|l|}{ Overall mortalitya) } \\
\hline Age & 1.021 & $1.007-1.035$ & 0.004 & 1.014 & $1.000-1.029$ & 0.046 \\
\hline Male sex & 1.076 & $0.835-1.386$ & 0.573 & - & - & - \\
\hline Smoking & 1.066 & $0.805-1.411$ & 0.655 & - & - & - \\
\hline Alcohol & 0.928 & $0.700-1.231$ & 0.606 & - & - & - \\
\hline Diabetes & 1.205 & $0.931-1.561$ & 0.157 & - & - & - \\
\hline CA 19-9 level & 1.010 & $1.006-1.014$ & $<0.001$ & 1.007 & $1.003-1.011$ & 0.001 \\
\hline Curative treatment & 0.345 & $0.266-0.447$ & $<0.001$ & 0.388 & $0.292-0.515$ & $<0.001$ \\
\hline $\mathrm{CT}+\mathrm{MR}$ vs. $\mathrm{CT}$ & 1.225 & $0.920-1.632$ & 0.164 & - & - & - \\
\hline Unresectable at MRI & 2.272 & $1.533-3.367$ & $<0.001$ & 1.500 & $0.981-2.294$ & 0.062 \\
\hline \multicolumn{7}{|l|}{ PDA recurrence ${ }^{b)}$} \\
\hline Age & 1.001 & $0.981-1.022$ & 0.891 & - & - & - \\
\hline Male sex & 1.417 & $0.965-2.079$ & 0.075 & - & - & - \\
\hline Smoking & 1.034 & $0.686-1.559$ & 0.873 & - & - & - \\
\hline Alcohol & 0.942 & $0.627-1.414$ & 0.771 & - & - & - \\
\hline Diabetes & 1.227 & $0.840-1.791$ & 0.290 & - & - & - \\
\hline CA 19-9 level & 1.004 & 0.993-1.016 & 0.448 & - & - & - \\
\hline Curative treatment & 1.292 & $0.876-1.907$ & 0.197 & - & - & - \\
\hline $\mathrm{CT}+\mathrm{MR}$ vs. CT & 0.947 & $0.636-1.412$ & 0.791 & - & - & - \\
\hline
\end{tabular}

The Cox proportional hazards model was used for all analyses. PDA, pancreas ductal adenocarcinoma; HR, hazard ratio; CI, confidence interval; CA 19-9, carbohydrate antigen 19-9; CT+MR, patients underwent both computed tomography and

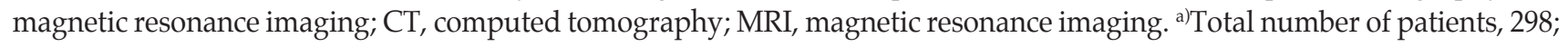
number of events, $245,{ }^{\mathrm{b}}$ Total number of patients who received curative surgical treatment, 161; number of events, 112.

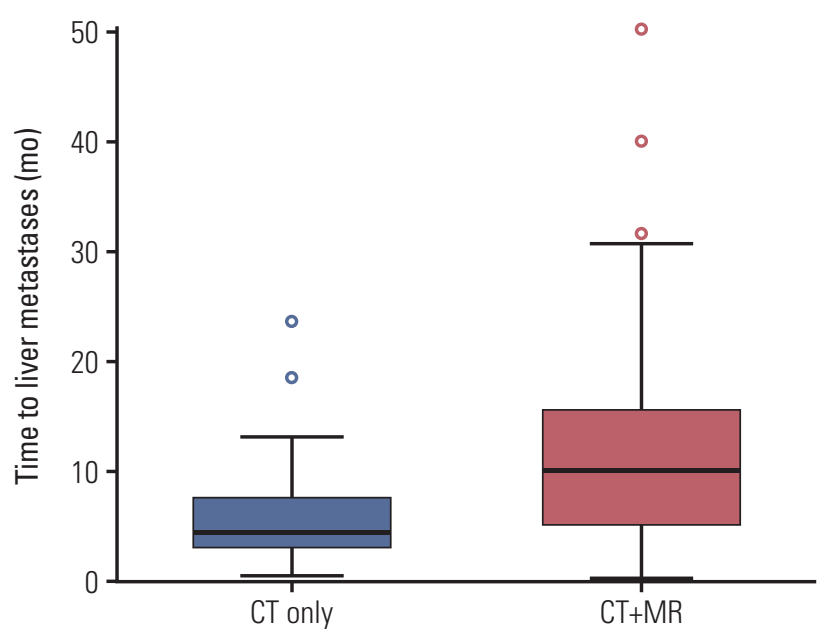

Fig. 4. Time to liver metastases in the computed tomography $(\mathrm{CT})$ only and the $\mathrm{CT}+\mathrm{MR}$ (patients underwent both $\mathrm{CT}$ and magnetic resonance imaging) groups of patients who received curative surgical treatment. liver metastases developed after curative surgery was 4.2 months in the CT group and 9.9 months in the $\mathrm{CT}+\mathrm{MR}$ group with significant difference between the two groups $(\mathrm{p}=0.011$ ), although the number of liver metastases confronted during their whole follow-up period was not very different (44 of 112 patients [39.3\%] in the CT+MR group and 20 of 49 patients [40.8\%] in the CT group) (Fig. 4).

\section{Discussion}

Our study results demonstrated that additional MRI resulted in changes of surgical resectability and treatment modifications in a significant proportion of patients who have potentially resectable states at $\mathrm{CT}$, owing to the additional detection of liver metastases $(n=16)$, major vascular invasion ( $n=13)$, enlarged para-aortic lymph node $(n=1)$, and peritoneal seeding $(\mathrm{n}=1)$.

The most common site of metastasis in pancreatic cancer is the liver $[13,14]$. A lot of studies have reported the superior 
sensitivity of MRI in detecting and characterizing liver lesions compared to $\mathrm{CT}$, even though most of them were performed in patients with colorectal cancer liver metastasis [15-18]. However, a recent large scale study on colorectal liver metastasis showed that there was no additional yield for MRI $(0 \%, 0 / 94)$ in the negative-liver-on-CT patients and only $3 \%(3 / 96)$ additional yield in patients with diminutive indeterminate hepatic lesions on $\mathrm{CT}$, suggesting that MRI might provide little clinical benefit in addition to $\mathrm{CT}$, in spite of the superior diagnostic accuracy of MRI than CT in these patients [19]. In our study on PDA, however, MRI identified occult liver metastases in $4.7 \%$ (9 of 191) of patients with negative-liver-on-CT and 35\% (7 of 20) of patients with diminutive indeterminate hepatic lesions on CT. A recently published study reported that $23.2 \%$ (16 of 69) of PDA patients with negative-liver-on-CT showed occult liver metastases on MRI [20]. These results suggest that MRI might provide additional clinical benefit for detecting synchronous liver metastasis in patients diagnosed with resectable PDA interpreted by CT (negative-liver-on-CT). It may be further supported by our results that showed a significant longer time of onset for hepatic metastases after curative surgery in patients of the CT+MR group ( 9.9 months) than in those of the CT only group (4.2 months).

Unlike liver metastases from colorectal cancer, there have been few published papers comparing the diagnostic performance of CT and MRI on liver metastases from PDA. A recent study reported the significantly higher sensitivity of gadoxetic acid-enhanced MRI (85\%) compared to CT (69\%) for detection of liver metastases from PDA, due to higher lesion-to-liver contrast on hepatocyte phase MR image [18]. A prospective pilot study with a small number of patients with liver metastases from PDA showed significantly higher sensitivity $(86.7 \%)$ and specificity $(97.5 \%)$ for MRI with DWI than multi-detector CT (MDCT) $(53.3 \%$ and $77.8 \%$, respectively) [21]. In our study, majority of patients $(86 \%, 186 / 216)$ underwent MRI using gadoxetic acid-enhanced MRI with DWI and these state-of-the-art MRI may be one of the reasons for the additional detection of liver metastases, resulting in changes in the treatment plan and longer time to liver metastases after surgery in the CT+MR group. Also, this pilot study showed that most liver metastases in patients with potentially resectable PDA on CT were small in size (mean diameter, $7.8 \pm 3.1 \mathrm{~mm}$; range 3 to $15 \mathrm{~mm}$ ) [21]. Hence, stateof-the-art MRI should be recommended [8].

The diagnostic performance of CT and MRI for evaluating vascular invasion is still controversial. The best performance was shown by MDCT in the past, but recent advances in MR technology has made the diagnostic performance of MDCT and MRI comparable (mean sensitivity, $80 \%$ vs. $65 \%$; mean specificity, $98 \%$ vs. $97 \%$, respectively) for vascular infiltration $[22,23]$. In our study, $6.0 \%$ of patients were reclassified from potentially resectable state to unresectable state due to additional detection of major vascular invasion by MRI in addition to CT. This may be contrary to the findings of previous studies demonstrating that $\mathrm{CT}$ was slightly better than or similar to MRI in the assessment of vasculature invasion. One reason for this discrepancy could be that most of the previous studies compared CT and MRI, but our study evaluated the additional value of MRI after CT, as in routine clinical practice.

In terms of overall and recurrence-free survival, there were no significant differences between the $\mathrm{CT}$ only and $\mathrm{CT}+\mathrm{MR}$ groups in our study. This was a retrospective study, and there are many unaccounted factors that affect the survival in pancreatic cancer, that include not only disease factors but also socioeconomic factors [24,25], and pre-treatment diagnostic imaging might not have direct effect on the survival. In our study, other well-known factors did not show significant differences between the $\mathrm{CT}$ only and $\mathrm{CT}+\mathrm{MR}$ groups except that higher proportion of patients belonging to the $\mathrm{CT}$ only group received the neoadjuvant therapy, and this might affect the clinical outcome $[3,24,26,27]$. Within the CT+MR group, patients with potentially resectable state had a higher overall survival than patients with unresectable state, suggesting that MRI allowed the clinicians to determine the most appropriate treatment.

The major limitation of our study was that there were no pre-defined criteria who should undergo additional MR imaging; this reflects the limitation in the current guideline and is the background for our study. Before analysis, we hypothesized that patients with borderline resectable or indeterminate states on CT were more likely to undergo MRI than patients with resectable state. However, there was no significant difference in proportion of the patients undergoing additional MRI among the three CT-based categories. Second, the imaging technique used in this study was not uniform. Some patients underwent single portal venous phase $\mathrm{CT}$ not the dual phase pancreatic protocol $\mathrm{CT}$, but there were no significant differences in proportion of $\mathrm{CT}$ protocols between the $\mathrm{CT}$ only and $\mathrm{CT}+\mathrm{MR}$ groups. In clinical practice, some patients undergo single phase $\mathrm{CT}$ (routine $\mathrm{CT}$ protocol) due to nonspecific symptoms, and we used it for evaluation of pancreatic cancer staging without obtaining dual phase CT because of radiation and reimbursement issues. Regarding MRI, some patients underwent MRI without DWI using a general extracellular contrast agent instead of the recently introduced hepatocyte-specific contrast agent. A recent paper showed no difference between the two contrast agents in the evaluation of liver metastases from colorectal cancer [28]. This is beyond the scope of our study, and a further study is needed. Finally, our study had the inherent limitations of a retrospective study.

In conclusion, additional MRI resulted in changes of 
resectability and treatment modification in a significant proportion of patients who had potentially resectable state on $\mathrm{CT}$ and delay in time to liver metastases in patients after curative surgery. Additional MRI to standard staging CT can be recommended for surgical candidates of PDA.

\section{Electronic Supplementary Material}

Supplementary materials are available at Cancer Research and Treatment website (https://www.e-crt.org).

\section{Conflicts of Interest}

Conflict of interest relevant to this article was not reported.

\section{References}

1. Siegel R, Ma J, Zou Z, Jemal A. Cancer statistics, 2014. CA Cancer J Clin. 2014;64:9-29.

2. Gillen S, Schuster T, Meyer Zum Buschenfelde C, Friess H, Kleeff J. Preoperative/ neoadjuvant therapy in pancreatic cancer: a systematic review and meta-analysis of response and resection percentages. PLoS Med. 2010;7:e1000267.

3. Kamisawa T, Wood LD, Itoi T, Takaori K. Pancreatic cancer. Lancet. 2016;388:73-85.

4. Orr RK. Outcomes in pancreatic cancer surgery. Surg Clin North Am. 2010;90:219-34.

5. McPhee JT, Hill JS, Whalen GF, Zayaruzny M, Litwin DE, Sullivan ME, et al. Perioperative mortality for pancreatectomy: a national perspective. Ann Surg. 2007;246:246-53.

6. Callery MP, Chang KJ, Fishman EK, Talamonti MS, William Traverso L, Linehan DC. Pretreatment assessment of resectable and borderline resectable pancreatic cancer: expert consensus statement. Ann Surg Oncol. 2009;16:1727-33.

7. Raman SP, Horton KM, Fishman EK. Multimodality imaging of pancreatic cancer-computed tomography, magnetic resonance imaging, and positron emission tomography. Cancer J. 2012;18:511-22.

8. Lee ES, Lee JM. Imaging diagnosis of pancreatic cancer: a state-of-the-art review. World J Gastroenterol. 2014;20:786477.

9. Tamburrino D, Riviere D, Yaghoobi M, Davidson BR, Gurusamy KS. Diagnostic accuracy of different imaging modalities following computed tomography (CT) scanning for assessing the resectability with curative intent in pancreatic and periampullary cancer. Cochrane Database Syst Rev. 2016;9:CD011515.

10. Egorov VI, Petrov RV, Solodinina EN, Karmazanovsky GG, Starostina NS, Kuruschkina NA. Computed tomographybased diagnostics might be insufficient in the determination of pancreatic cancer unresectability. World J Gastrointest Surg. 2013;5:83-96.

11. Kim YE, Park MS, Hong HS, Kang CM, Choi JY, Lim JS, et al. Effects of neoadjuvant combined chemotherapy and radiation therapy on the CT evaluation of resectability and staging in patients with pancreatic head cancer. Radiology. 2009;250:75865.

12. National Comprehensive Cancer Network. Practice guidelines in oncology for pancreatic adenocarcinoma V2 [Internet]. Fort Washington, PA: National Comprehensive Cancer Network; 2015 [cited 2018 Feb 5]. Available from: http:// www.nccn.org. professionals/physician_PDF/pancreatic.pdf.

13. Kamisawa T, Isawa $T$, Koike $M$, Tsuruta $K$, Okamoto A. Hematogenous metastases of pancreatic ductal carcinoma. Pancreas. 1995;11:345-9.

14. Disibio G, French SW. Metastatic patterns of cancers: results from a large autopsy study. Arch Pathol Lab Med. 2008;132: 931-9.

15. Kim HJ, Lee SS, Byun JH, Kim JC, Yu CS, Park SH, et al. Incremental value of liver MR imaging in patients with potentially curable colorectal hepatic metastasis detected at CT: a prospective comparison of diffusion-weighted imaging, gadoxetic acid-enhanced MR imaging, and a combination of both MR techniques. Radiology. 2015;274:712-22.

16. Scharitzer M, Ba-Ssalamah A, Ringl H, Kolblinger C, Grunberger T, Weber M, et al. Preoperative evaluation of colorectal liver metastases: comparison between gadoxetic acid-enhanced 3.0-T MRI and contrast-enhanced MDCT with histopathological correlation. Eur Radiol. 2013;23:2187-96.

17. Muhi A, Ichikawa T, Motosugi U, Sou H, Nakajima H, Sano $\mathrm{K}$, et al. Diagnosis of colorectal hepatic metastases: comparison of contrast-enhanced CT, contrast-enhanced US, superparamagnetic iron oxide-enhanced MRI, and gadoxetic acidenhanced MRI. J Magn Reson Imaging. 2011;34:326-35.

18. Motosugi U, Ichikawa T, Morisaka H, Sou H, Muhi A, Kimura $\mathrm{K}$, et al. Detection of pancreatic carcinoma and liver metastases with gadoxetic acid-enhanced MR imaging: comparison with contrast-enhanced multi-detector row CT. Radiology. 2011; 260:446-53.

19. Han K, Park SH, Kim KW, Kim HJ, Lee SS, Kim JC, et al. Use of liver magnetic resonance imaging after standard staging abdominopelvic computed tomography to evaluate newly diagnosed colorectal cancer patients. Ann Surg. 2015;261: 480-6.

20. Chew C, O'Dwyer PJ. The value of liver magnetic resonance imaging in patients with findings of resectable pancreatic cancer on computed tomography. Singapore Med J. 2016;57: 334-8.

21. Holzapfel K, Reiser-Erkan C, Fingerle AA, Erkan M, Eiber MJ, 
Rummeny EJ, et al. Comparison of diffusion-weighted MR imaging and multidetector-row CT in the detection of liver metastases in patients operated for pancreatic cancer. Abdom Imaging. 2011;36:179-84.

22. Koelblinger C, Ba-Ssalamah A, Goetzinger P, Puchner S, Weber M, Sahora K, et al. Gadobenate dimeglumine-enhanced 3.0-T MR imaging versus multiphasic 64-detector row CT: prospective evaluation in patients suspected of having pancreatic cancer. Radiology. 2011;259:757-66.

23. Chen FM, Ni JM, Zhang ZY, Zhang L, Li B, Jiang CJ. Presurgical evaluation of pancreatic cancer: a comprehensive imagingcomparison of CT versus MRI. AJR Am J Roentgenol. 2016; 206:526-35.

24. Bilici A. Prognostic factors related with survival in patients with pancreatic adenocarcinoma. World J Gastroenterol. 2014;20:10802-12.
25. El-Rayes BF, Jasti P, Severson RK, Almhanna K, Philip PA, Shields A, et al. Impact of race, age, and socioeconomic status on participation in pancreatic cancer clinical trials. Pancreas. 2010;39:967-71.

26. Kim SM, Eads JR. Adjuvant and neoadjuvant therapy for resectable pancreatic and periampullary cancer. Surg Clin North Am. 2016;96:1287-300.

27. O'Reilly EM, Perelshteyn A, Jarnagin WR, Schattner M, Gerdes $\mathrm{H}$, Capanu $\mathrm{M}$, et al. A single-arm, nonrandomized phase II trial of neoadjuvant gemcitabine and oxaliplatin in patients with resectable pancreas adenocarcinoma. Ann Surg. 2014;260: $142-8$.

28. Seo N, Park MS, Han K, Lee KH, Park SH, Choi GH, et al. Magnetic resonance imaging for colorectal cancer metastasis to the liver: comparative effectiveness research for the choice of contrast agents. Cancer Res Treat. 2018;50:60-70. 